DOI: https://doi.org/10.17816/psaic614

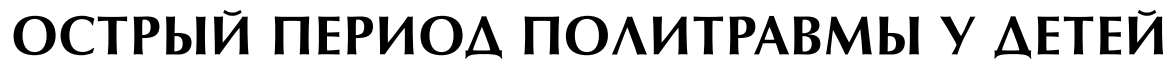 В СВЕТЕ АИСКРИМИНАНТНОГО АНААИЗА
}

(С В.Б. Баковский ${ }^{1}$, С.И. Головкин ${ }^{2}$, Т.в. Кухарова ${ }^{3}$, В.А. Уткин ${ }^{4}$, Е.Н. Чалая ${ }^{4}$, Н.А. Шабалдин ${ }^{2}$

${ }^{1}$ Государственное автономное учреждение здравоохранения «Кузбасская областная детская клиническая больница», Кемерово;

${ }^{2}$ Федеральное государственное бюджетное образовательное учреждение высшего образования «Кемеровский государственный медицинский университет» Министерства здравоохранения Российской Федерации, Кемерово;

${ }_{3}^{3}$ Федеральное государственное автономное образовательное учреждение высшего образования «Санкт-Петербургский государственный университет аэрокосмического приборостроения», Санкт-Петербург;

${ }^{4}$ Пятигорский научно-исследовательский институт курортологии - филиал Федерального государственного бюджетное учреждения «Северо-Кавказский научно-клинический центр Федерального медико-биологического агентства России в г. Пятигорске», Пятигорск

- Аля цитирования: Баковский В.Б., Головкин С.И., Кухарова Т.В., Уткин В.А., Чалая Е.Н., Шабалдин Н.А. Острый периол политравмы у Аетей в свете Аискриминантного анализа // Российский вестник Аетской хирургии, анестезиологии и реаниматологии. - 2020. - Т. 10. № 2. - C. 145-156. DOI: https://doi.org/10.17816/psaic614

Поступи^а: 15.01 .2020

ОАобрена: 11.02 .2020

Опубликована: 12.03 .2020

Введение. В лечении политравмы у детей актуально не только выявить признаки, характеризующие тяжесть острого периода, но и выразить количественно приоритеты параметров, в совокупности отражающих тенденции динамики ведущих патофизиологических проявлений на каждом этапе реализации программы выведения пациента из состояния тяжелого шока.

Цель. С применением дискриминантного анализа получить количественное отображение реконвалесцентной или танатогенной направленности динамики политравмы у детей в первые дни преодоления ее последствий, представив это в виде векторов с коэффициентами, выражающими приоритеты отбираемых по ходу лечения данных анализов, и, исходя из патогенетически обоснованного представления о роли каждого из них, уточнить тактику врача на каждом фиксируемом отрезке времени. Материалы и методы. Был проведен анализ данных 45 детей с политравмой в возрасте от 2,5 до 17 лет, госпитализированных в реанимационное отделение ГАУЗ КО ОДКБ г. Кемерово. По гендерному составу - 34 мальчика и 11 девочек. С применением дискриминантного анализа проанализированы две группы, где в первой - выжившие, во второй - умершие. В обеих доминировала тяжелая черепно-мозговая травма. Для клинической оценки тяжести травмы использовали шкалу ISS. Результаты. По совокупности с объективно полученными данными о структуре политравмы дрейф в направлении благополучного исхода определяется в целом и на границах «день ото дня» приоритетами по калию, pН, белой крови и гематокриту, а для вектора танатогенной направленности характерна картина нарастающей полиорганной недостаточности, где, несмотря на своевременно выполненные оперативные вмешательства по остановке внутреннего кровотечения, весьма активные усилия по компенсации гиповолемии, ацидоза и использование адекватных средств детоксикации, ухудшение в состоянии ребенка проявляет себя нарастающими потерями по калию на фоне практически отсутствующей реакции со стороны лейкоцитов. Выводы. С применением дискриминантного анализа становится возможным полнее раскрыть особенности многомерной динамики проявлений политравмы у детей в первые дни реанимационных мероприятий, численно выразить приоритеты отдельных параметров, характеризующих их состояние, и, сообразно степени тяжести и индивидуальной реакции пациента, в реальном времени оптимизировать тактику лечения.

Ключевые слова: политравма; структура; дети; дискриминантный анализ.

\section{Комментарий редакиии!}

Инструменты дискриминантного анализа позволяют более точно объективизировать тяжесть состояния пациента и соответственно корригировать его лечение, но при интерпретации выявленных закономерностей необходимо использовать более понятное изложение, учитьвая иелевую аудиторию журнала. Приведение примера использования вычисленных коэфбициентов на каком-либо клиническом наблюдении позволило бы повысить практическую значимость полученных результатов. 


\title{
ACUTE PERIOD OF POLYTRAUMA IN CHILDREN IN THE LIGHT OF DISCRIMINANT ANALYSIS
}

\author{
(C) V.B. Bakovsky ${ }^{1}$, S.I. Golovkin ${ }^{2}$, T.V. Kukharova ${ }^{3}$, V.A. Utkin ${ }^{4}$, E.N. Chalaya ${ }^{4}$, \\ N.A. Shabaldin $2 \bowtie$ \\ ${ }^{1}$ Children's Regional Clinical Hospital, Kemerovo, Russia; \\ ${ }^{2}$ Kemerovo State Medical University, Kemerovo, Russia; \\ ${ }^{3}$ Saint Petersburg State University of Aerospace Instrumentation, Saint Petersburg, Russia; \\ ${ }^{4}$ Pyatigorsk State Research Institute of Balneology of the Federal medical and biological Agency, \\ Pyatigorsk, Russia
}

- For citation: Bakovsky VB, Golovkin SI, Kukharova TV, Utkin VA, Chalaya EN, Shabaldin NA. Acute period of polytrauma in children in the light of discriminant analysis. Russian J oumal of Pediatric Surgery, Anesthesia and Intensive Care. 2020;10(2):145-156. DOl: https://doi.org/10.17816/ psaic614

Introduction. The treatment of polytrauma in children requires identifying the signs that characterize the severity of the acute period and quantifying the priorities of the parameters. Collectively, these reflect the direction of drift of the leading pathophysiological manifestations at each stage of the patient withdrawal program from a state of severe shock.

Purpose. This study uses discriminant analysis to clarify the tactics of children with polytrauma in the first days of overcoming its consequences. It is based on the pathogenetically sound idea that each of the observed parameter's role, together in the form of a vector, reflects injury severity and the child's prognosis.

Materials and methods. This analysis included 45 children (34 boys and 11 girls) with polytrauma aged from 2.5 to 17 years and hospitalized in Kemerovo's intensive care unit. Two groups were analyzed: the survivors and those who were deceased. Both were dominated by severe traumatic brain injury (PMT). The injury severity score (ISS) scale was used for clinical assessment of injury severity.

Results. Combined with objectively obtained data on the structure of polytrauma in the direction of drift, a successful outcome is defined as a whole. It borders on the "day to day" priorities, potassium, $\mathrm{PH}$, white blood count, and hematocrit. Also, the vector orientation pattern was observed to increase organ failure. This progressive decline occurred despite timely surgical intervention to stop internal bleeding, very active efforts to compensate for hypovolemia, acidosis, and the use of adequate means of detoxification. The deterioration in the child's condition manifests itself by increased potassium losses against the background of almost no reaction from leukocytes.

Conclusions. The application of discriminant analysis enables the better revelation of the peculiarities of a polytrauma's multidimensional dynamics in children in the first few days of resuscitation. It also permits the numerical expression of the priorities of individual parameters that describe their state, and by the severity and individual patient response in real-time to optimize treatment.

Keywords: polytrauma; structure; children; discriminant analysis.

\section{BBEАЕНИЕ}

Лечение политравмы сопряжено с большими трудностями $[1,2]$. Они возникают и при оказании помощи детям с политравмой на догоспитальном этапе и в период стационарного лечения травматического шока $[3,4]$. Не вызывает сомнений тот факт, что объективно анализировать патологические процессы без должного структурирования политравмы практически невозможно [5]. Однако предметное структурирование политравмы у детей чаще всего затруднено, как в силу многогранности ее проявлений, так и из-за необходимости срочного принятия решений на ограниченном отрезке времени. Вполне очевидно, что это отражается и на качестве реализации неотложных мер.

В работе [6], предваряющей данное исследование, нами показано, что с применением шкалы тяжести травмы (Injury Severity Score - ISS), которая и у детей является золотым стандартом [7], удается объективно и с достаточной надежностью выделить в группах травмированных пациентов признаки, характеризующие тяжесть острого периода, оптимизировать схему обследования и программу лечения. Но при этом еще бо- 
лее важным оказывается не только их выявить, но и выразить количественно приоритеты параметров, в совокупности в виде вектора отражающих реконвалесцентную или танатогенную направленность динамики ведущих патофизиологических проявлений на каждом этапе реализации программы выведения пациента из состояния тяжелого шока.

Цель работы - с применением дискриминантного анализа получить количественное отображение реконвалесцентной или танатогенной направленности динамики политравмы у детей в первые дни преодоления ее последствий, представив это в виде векторов с коэффициентами, выражающими приоритеты отбираемых по ходу лечения данных анализов, и, исходя из патогенетически обоснованного представления о роли каждого из них, уточнить тактику врача на каждом фиксируемом отрезке времени.

\section{МАТЕРИААЫ И МЕТОАЫ}

Выполнен анализ сплошной выборки 45 детей с политравмой в возрасте от 2,5 до 17 лет, госпитализированных в реанимационное отделение ГАУЗ КО «Областная детская клиническая больница» (г. Кемерово) за период с 2014 по 2019 г. По гендерному составу - 34 мальчика и 11 девочек. В основном, в анализируемой группе были дети старшего возраста - от 13 до 17 лет - 34 (75,6 \%) из 45 пациентов. Критерием включения был установленный диагноз политравмы, то есть множественная либо сочетанная тяжелая травма, состояние травматического шока при поступлении и оценка тяжести травмы по шкале ISS не менее 16 баллов. Из исследования исключены данные пациентов, умерших до истечения четырех суток с момента поступления.

В первую самую многочисленную группу детей (выжившие пациенты) вошли 36 (80 \%) из 45 пострадавших с доминирующей тяжелой черепно-мозговой травмой (ТЧМТ), причем у 5 (13,9\%) пострадавших в этой группе диагностирована открытая черепно-мозговая травма (ОЧМТ), у 2 (5,5 \%) - диффузные аксональные повреждения мозга (ДАП).

Вторая группа - это 9 умерших детей, что составило $20 \%$ от общего числа проанализированных наблюдений. В нее вошли 4 ребенка, у которых доминировала ТЧМТ с наличием тяжелой скелетной травмы (СТ), и 5 пациентов с ТЧМТ и повреждением внутренних органов (ПВО) с синдромом внутрибрюшного кровотечения.

Всем пострадавшим проводили общеклинические и биохимические анализы крови и мочи для интегральной оценки состояния больного, жизнеобеспечения головного мозга, почек и системного кровообращения. По показаниям выполнены экстренные операции по остановке внутрибрюшного кровотечения (5 детей). Для оценки тяжести травмы использовалась шкала ISS.

Анализ наблюдений выполнен с использованием стандартного пакета Microsoft Office Excel 2003 с подключением надстройки AtteStat v.8.0 и модулей библиотеки SADQO на языке PASCAL. Проанализированы данные за первые 4 сут наблюдений по 22 показателям отбираемых анализов (полный перечень не приводится), из которых методом статистических испытаний (Монте-Карло) были отобраны 7 параметров крови, показавших наиболее эффективное разграничение этапов наблюдения с применением дискриминантного анализа.

\section{РЕЗУАЬТАТЫ ИССАЕАОВАНИЯ И ИХ ОБСУЖАЕНИЕ}

Клинически в обеих группах доминировала ТЧМТ с баллами по шкале ISS от 45 до 75 баллов. В табл. 1 представлены данные по исследуемым показателям в первые сутки травмы, где видно, что значимые различия между группами были выявлены для уровня гемоглобина, он был ниже в группе умерших; концентрации ионов натрия в периферической крови, показатель был выше в группе умерших; и для бального показателя ISS, который также был выше в группе умерших.

Вариационные характеристики наблюдений, как по выжившим (табл. 2), так и по умершим (табл. 3) пациентам, непосредственно и в сопоставлениях, конструктивно значимой информации для интенсивно загруженного в первые дни реанимационных мероприятий травматолога, на наш взгляд, должного объема и интереса не представляют.

В самом деле, по выжившим пациентам отмеченные в 1-й день отклонения от нормального распределения по лейкоцитам и натрию, а также по калию и натрию на 2-й день, или по рН на 2-й и 4-й день, носят скорее манифестный характер. 
Сравнительная характеристика групп выживших и умерших пациентов по исследуемым показателям

Comparative characteristics for groups of survivors and deceased patients according to the studied indicators

\begin{tabular}{|c|c|c|c|c|c|c|c|}
\hline \multirow{2}{*}{ Показатель } & \multicolumn{3}{|c|}{ Выжившие, $n=36$} & \multicolumn{3}{|c|}{ Умершие, $n=9$} & \multirow{2}{*}{$p$} \\
\hline & Me & $Q_{25}$ & $Q_{75}$ & Me & $Q_{25}$ & $Q_{75}$ & \\
\hline $\mathrm{Hb}$, г/л & 120,56 & 147,00 & 94,13 & 103,67 & 128,41 & 78,93 & $0,01^{*}$ \\
\hline $\mathrm{Ht}, \%$ & 35,63 & 42,64 & 28,62 & 34,57 & 46,05 & 23,09 & $>0,05$ \\
\hline $\mathrm{Er}, \times 10^{12} /$ л & 3,72 & 4,61 & 2,82 & 4,32 & 5,44 & 3,21 & $>0,05$ \\
\hline Le, $\times 10^{9} /$ л & 14,32 & 23,72 & 4,93 & 12,96 & 19,95 & 5,96 & $>0,05$ \\
\hline $\mathrm{pH}$ & 7,40 & 7,64 & 7,15 & 7,26 & 7,45 & 7,08 & $>0,05$ \\
\hline $\mathrm{K}^{+}$, ммоль/л & 3,79 & 5,06 & 2,53 & 4,24 & 5,38 & 3,10 & $>0,05$ \\
\hline $\mathrm{Na}^{+}$, ммоль/л & 137,08 & 162,26 & 111,91 & 158,28 & 179,41 & 137,14 & $0,007^{\star}$ \\
\hline ISS & 23,22 & 31,15 & 15,29 & 39,44 & 44,81 & 34,08 & $0,004^{*}$ \\
\hline
\end{tabular}

* Значимые различия. * Significant differences.

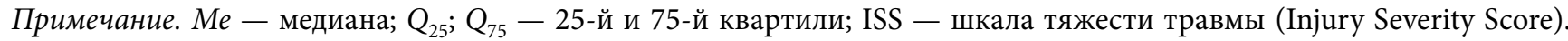
Note. $M e-$ Median Value, $Q_{25} ; Q_{75}-25^{\text {th }}$ and $75^{\text {th }}$ quartiles, ISS - Injury Severity score

Таблица 2 / Table 2

\section{Вариационные характеристики наблюдений у выживших пациентов}

Variational observational characteristics for surviving patients

\begin{tabular}{|c|c|c|c|c|c|c|c|c|}
\hline \multicolumn{2}{|r|}{ Параметры } & $\mathrm{Hb}$, г/л & $\mathrm{Ht}, \%$ & $\mathrm{Er}, \times 10^{12} /$ л & Le, $\times 10^{9} /$ л & $\mathrm{pH}$ & $\begin{array}{c}\mathrm{K}^{+}, \\
\text {ммоль/л }\end{array}$ & $\begin{array}{c}\mathrm{Na}^{+}, \\
\text {ммоль/л }\end{array}$ \\
\hline \multirow{9}{*}{$\begin{array}{l}1-\text { й } \\
\text { день }\end{array}$} & $\min$ & 89 & 24,2 & 3,01 & 4,4 & 7,114 & 1,46 & 135 \\
\hline & $\max$ & 151 & 41 & 5,01 & 54,9 & 7,52 & 3,61 & 149,2 \\
\hline & Размах & 62 & 16,8 & 2,00 & 50,5 & 0,406 & 2,15 & 14,2 \\
\hline & Среднее $(M)$ & 111,3 & 31,41 & 3,972 & 18,21 & 7,335 & 2,898 & 139,27 \\
\hline & Ошибка $( \pm m)$ & 5,4 & 1,57 & 0,189 & 3,67 & 0,033 & 0,167 & 1,081 \\
\hline & Коэффициент вариации & 0,169 & 0,173 & 0,164 & 0,698 & 0,016 & 0,2 & 0,027 \\
\hline & Асимметрия & нет & нет & нет & $p<0,001$ & нет & нет & $p<0,02$ \\
\hline & Эксцесс & нет & нет & нет & $p<0,001$ & нет & нет & $p<0,02$ \\
\hline & $\begin{array}{l}\text { Отклонение от N - } \\
\text { распределение по Джири }\end{array}$ & нет & нет & нет & $p<0,001$ & нет & нет & $p<0,015$ \\
\hline \multirow{9}{*}{$\begin{array}{l}2 \text {-й } \\
\text { день }\end{array}$} & $\min$ & 89 & 25 & 3,25 & 4,1 & 7,248 & 0,395 & 135 \\
\hline & $\max$ & 145 & 42 & 4,79 & 26,2 & 7,582 & 4,71 & 151,8 \\
\hline & Размах & 56 & 17 & 1,54 & 22,1 & 0,334 & 4,315 & 16,8 \\
\hline & Среднее $(M)$ & 111,92 & 32,03 & 3,947 & 12,79 & 7,377 & 3,004 & 139,38 \\
\hline & Ошибка $( \pm m)$ & 4,51 & 1,25 & 0,126 & 1,65 & 0,026 & 0,299 & 1,28 \\
\hline & Коэффициент вариации & 0,14 & 0,135 & 0,111 & 0,448 & 0,012 & 0,345 & 0,032 \\
\hline & Асимметрия & нет & нет & нет & нет & нет & нет & $p<0,003$ \\
\hline & Эксцесс & нет & нет & нет & нет & нет & $p<0,05$ & $p<0,001$ \\
\hline & $\begin{array}{l}\text { Отклонение от } \mathrm{N}- \\
\text { распредение по Джири }\end{array}$ & нет & нет & нет & $p<0,054$ & $p<0,053$ & $p<0,001$ & $p<0,001$ \\
\hline
\end{tabular}


Продолжение табл. 2 / Continuation of Table 2

\begin{tabular}{|c|c|c|c|c|c|c|c|c|}
\hline \multicolumn{2}{|r|}{ Параметры } & $\mathrm{Hb}$, г/л & $\mathrm{Ht}, \%$ & $\mathrm{Er}, \times 10^{12} /$ л & Le, $\times 10^{9}$ /л & $\mathrm{pH}$ & $\begin{array}{c}\mathrm{K}^{+}, \\
\text {ммоль/л }\end{array}$ & $\begin{array}{c}\mathrm{Na}^{+}, \\
\text {ммоль/л }\end{array}$ \\
\hline \multirow{9}{*}{$\begin{array}{l}\text { 3-й } \\
\text { день }\end{array}$} & $\min$ & 92 & 26 & 3,41 & 3,7 & 7,289 & 3,08 & 134 \\
\hline & $\max$ & 143 & 39 & 4,75 & 23,3 & 7,44 & 4,9 & 152,6 \\
\hline & Размах & 51 & 13 & 1,34 & 19,6 & 0,151 & 1,82 & 18,6 \\
\hline & Среднее $(M)$ & 112,8 & 32,47 & 4,028 & 11,44 & 7,381 & 3,83 & 141,28 \\
\hline & Ошибка $( \pm m)$ & 5,58 & 1,41 & 0,146 & 1,75 & 0,013 & 0,138 & 1,74 \\
\hline & Коэффициент вариации & 0,171 & 0,151 & 0,125 & 0,53 & 0,006 & 0,125 & 0,043 \\
\hline & Асимметрия & нет & нет & нет & нет & нет & нет & нет \\
\hline & Эксцесс & нет & нет & нет & нет & нет & нет & нет \\
\hline & $\begin{array}{l}\text { Отклонение от } \mathrm{N}- \\
\text { распредение по Джири }\end{array}$ & нет & нет & нет & нет & нет & нет & нет \\
\hline \multirow{9}{*}{$\begin{array}{l}\text { 4-й } \\
\text { день }\end{array}$} & $\min$ & 84 & 23 & 3,08 & 5,3 & 7,324 & 3,12 & 134 \\
\hline & $\max$ & 159 & 44,9 & 5,56 & 14,5 & 7,485 & 3,89 & 149 \\
\hline & Размах & 75 & 21,9 & 2,48 & 9,2 & 0,161 & 0,77 & 15 \\
\hline & Среднее $(M)$ & 113,5 & 32,88 & 3,967 & 10,1 & 7,412 & 3,505 & 140,24 \\
\hline & Ошибка $( \pm m)$ & 6,1 & 1,69 & 0,213 & 0,93 & 0,013 & 0,073 & 1,28 \\
\hline & Коэффициент вариации & 0,186 & 0,178 & 0,186 & 0,32 & 0,006 & 0,072 & 0,032 \\
\hline & Асимметрия & нет & нет & нет & нет & нет & нет & нет \\
\hline & Эксцесс & нет & нет & нет & нет & нет & нет & нет \\
\hline & $\begin{array}{l}\text { Отклонение от } \mathrm{N}- \\
\text { распределение по Джири }\end{array}$ & нет & нет & нет & нет & $p<0,051$ & нет & нет \\
\hline
\end{tabular}

А с применением дисперсионного анализа по Фишеру уловить неоднородности по гемоглобину, гематокриту и числу эритроцитов $(p<0,015)$ удается только лишь по контингенту, а не на срезе времени.

В отношении же числа лейкоцитов по выжившим, неоднородность выявлена и по пациентам и по времени $(p<0,04)$, причем с явно выраженной акцентуацией к 1-му дню $(p<0,015)$. Примерно так же выглядит и динамика значений $\mathrm{pH}(p<0,05)$, где чуть более контрастны отличия еще и в сравнении каждого дня с каждым $(p<0,05)$. И более отчетливо $(p<0,005)$ это проявляется по калию. А по натрию неоднородность на срезе времени значима не хуже $p<0,002$.

По умершим значимый $(p<0,05)$ эксцесс по гемоглобину на 3 -й день и по натрию $(p<0,015)$ на 4 -й день, как и отмеченное на 4-й день отклонение от нормального распределения по калию $(p<0,002)$, по-видимому, всего лишь отражает отклик на внешнюю активность в отношении пациента. А в свете дисперсионного анализа по Фишеру и межэтапных попарных сопоставлений по каждому параметру позитивной динамики не усматривается. Отмечена лишь неоднородность в индивидуальных проявлениях по лейкоцитам $(p<0,001)$ и натрию $(p<0,001)$. И отличия в попарных сопоставлениях 2-го и 3-го дня с 4-м по калию $(p<0,025)$, как и в сравнении 3 -го и 4-го дня с 1-м по натрию $(p<0.05)$, возможно, тоже отражают лишь результат внешней по отношению к пациентам активности.

Ранее мы обращали внимание $[8,9]$, что основанные на патогенетических представлениях медицинские классификации контекстно, как правило, скрывают внутри себя фактор времени, и в наблюдениях состояния пациента, определяемых дискретно в последовательности от «здоровых» к «наиболее тяжелым» не следует полагать возможным мгновенный переход в том же или в противоположном направлении, минуя промежуточные этапы. И, когда надежно диагностируемые состояния могут быть разграничены исходя из достаточно полных статистических выборок с применением методов дискриминантного анализа и представлены в форме канонических дискриминантных 
Вариационные характеристики наблюдений по умершим пациентам

Таблица 3 / Table 3

Variational observational characteristics for deceased patients

\begin{tabular}{|c|c|c|c|c|c|c|c|c|}
\hline \multicolumn{2}{|r|}{ Параметры } & $\mathrm{Hb}$, г/л & $\mathrm{Ht}, \%$ & $\mathrm{Er}, \times 10^{12} /$ л & Le, $\times 10^{9} / \pi$ & $\mathrm{pH}$ & $\begin{array}{c}\mathrm{K}^{+}, \\
\text {ммоль/л }\end{array}$ & $\begin{array}{c}\mathrm{Na}^{+}, \\
\text {ммоль/л }\end{array}$ \\
\hline \multirow{9}{*}{$\begin{array}{l}\text { 1-й } \\
\text { день }\end{array}$} & $\min$ & 87 & 23 & 3 & 6,1 & 7,334 & 1,43 & 135 \\
\hline & $\max$ & 128 & 34 & 4,64 & 23,5 & 7,591 & 3,47 & 158 \\
\hline & Размах & 41 & 11 & 1,64 & 17,4 & 0,257 & 2,04 & 23 \\
\hline & Среднее $(M)$ & 109,6 & 30,8 & 4,028 & 13,12 & 7,43 & 2,782 & 149,3 \\
\hline & Ошибка $( \pm m)$ & 7,2 & 2,12 & 0,286 & 3,14 & 0,046 & 0,383 & 3,92 \\
\hline & Коэффициент вариации & 0,147 & 0,158 & 0,159 & 0.535 & 0,014 & 0,308 & 0,059 \\
\hline & Асимметрия & нет & нет & нет & нет & нет & нет & нет \\
\hline & Эксцесс & нет & нет & нет & нет & нет & нет & нет \\
\hline & $\begin{array}{l}\text { Отклонение от N - pac- } \\
\text { пределение по Джири }\end{array}$ & нет & нет & нет & нет & нет & нет & нет \\
\hline \multirow{9}{*}{$\begin{array}{l}\text { 2-й } \\
\text { день }\end{array}$} & $\min$ & 94 & 29 & 3,55 & 6,2 & 7,236 & 2,62 & 146 \\
\hline & $\max$ & 127 & 39,1 & 4,54 & 15,4 & 7,48 & 4,74 & 186 \\
\hline & Размах & 33 & 10,1 & 0,99 & 9,2 & 0,244 & 2,12 & 40 \\
\hline & Среднее $(M)$ & 114,4 & 33,18 & 4,05 & 10,91 & 7,368 & 3,328 & 161,8 \\
\hline & Ошибка $( \pm m)$ & 6,1 & 1,83 & 0,175 & 1,57 & 0,042 & 0,376 & 6,9 \\
\hline & Коэффициент вариации & 0,119 & 0,123 & 0,097 & 0,321 & 0,013 & 0,252 & 0,095 \\
\hline & Асимметрия & нет & нет & нет & нет & нет & нет & нет \\
\hline & Эксцесс & нет & нет & нет & нет & нет & нет & нет \\
\hline & $\begin{array}{l}\text { Отклонение от } \mathrm{N}-\text { рас- } \\
\text { пределение по Джири }\end{array}$ & нет & нет & нет & нет & нет & нет & нет \\
\hline \multirow{9}{*}{$\begin{array}{l}\text { 3-й } \\
\text { день }\end{array}$} & $\min$ & 95 & 28,3 & 3,58 & 6,6 & 7,228 & 2,92 & 147 \\
\hline & $\max$ & 147 & 48 & 5,99 & 22,9 & 7,47 & 5,58 & 178 \\
\hline & Размах & 52 & 19,7 & 2,41 & 16,3 & 0,242 & 2,66 & 31 \\
\hline & Среднее $(M)$ & 120,4 & 35,36 & 4,444 & 12,48 & 7,334 & 4,228 & 158,2 \\
\hline & Ошибка $( \pm m)$ & 9,52 & 3,62 & 0,462 & 2,88 & 0,041 & 0,453 & 5,5 \\
\hline & Коэффициент вариации & 0,177 & 0,229 & 0,232 & 0,516 & 0,013 & 0,24 & 0,078 \\
\hline & Асимметрия & нет & нет & нет & нет & нет & нет & нет \\
\hline & Эксцесс & $p<0,05$ & нет & нет & нет & нет & нет & нет \\
\hline & $\begin{array}{l}\text { Отклонение от N - pac- } \\
\text { пределение по Джири }\end{array}$ & нет & нет & нет & нет & нет & нет & нет \\
\hline \multirow{9}{*}{$\begin{array}{l}4 \text {-й } \\
\text { день }\end{array}$} & $\min$ & 102 & 28,7 & 3,62 & 9,3 & 7,26 & 3,44 & 146 \\
\hline & $\max$ & 139 & 47 & 5,6 & 16,5 & 7,461 & 4,13 & 176 \\
\hline & Размах & 37 & 18,3 & 1,98 & 7,2 & 0,201 & 0,69 & 30 \\
\hline & Среднее $(M)$ & 118 & 35,06 & 4,446 & 11,36 & 7,37 & 3,796 & 159,9 \\
\hline & Ошибка $( \pm m)$ & 6,3 & 3,25 & 0,339 & 1,31 & 0,036 & 0,109 & 6,6 \\
\hline & Коэффициент вариации & 0,119 & 0,207 & 0,17 & 0,257 & 0,011 & 0,064 & 0,093 \\
\hline & Асимметрия & нет & нет & нет & нет & нет & нет & нет \\
\hline & Эксцесс & нет & нет & нет & нет & нет & нет & $p<0,015$ \\
\hline & $\begin{array}{l}\text { Отклонение от N - pac- } \\
\text { пределение по Джири }\end{array}$ & нет & нет & нет & нет & нет & $p<0,002$ & нет \\
\hline
\end{tabular}


или же простых классифицирующих функций $[10,11]$ становится возможным ими воспользоваться, чтобы не только помочь врачу соответствующими номограммами автоматизируемой диагностики [12], но и представить динамику конкретных патологических изменений в организме, полагая параметры соответствующих наблюдений распределенными от здорового до тяжелейшего случая сообразно отсчетам в определенном смысле «условной» шкалы времени [9].

В данной работе рассмотрена возможность применения подобного подхода на срезе уже реального, а не условного времени, когда необходимы интенсивные вмешательства, влекущие значимые изменения состояния пациента в масштабе первых дней преодоления последствий политравмы у детей.

Напомним, что в основу дискриминантного анализа положены представления о возможности разграничить состояния пациента, воспользовавшись или каноническими дискриминантными или же так называемыми простыми классифицирующими функциями $[13,14]$. И если, опираясь на первые можно доказательно оценить качество разграничения или состоятельность классификации и при этом ранжировать параметры наблюдений по их диагностической значимости [11], то последние в контексте данного исследования представляют особый интерес в свете возможности установить тенденции «дрейфа» патологии в танатогенном или же в реконвалесцентном направлении. Полагая, что и в первые минуты, и в первые часы, и в первые дни борьбы за жизнь и здоровье ребенка совокупность описывающих его состояние параметров на каждый следующий момент наблюдения отражает значимые отличия последующего образа патологии от предыдущего, мы воспользовались методами дискриминантного анализа [10] в сопоставлении данных 1, 2, 3 и 4-го дней наблюдений в условиях реанимационной палаты.

В доступных нам источниках мы не обнаружили сведений о применении такого подхода применительно к наблюдениям при интенсивных вмешательствах и, полагая, что такая гипотеза выдвигается, и такой подход применяется впервые, наверное, не следовало ожидать высокого контраста данных и высокой значимости их динамики. Тем не менее значимость первой канонической дискриминантной функции по выжившим пациентам оказалась не хуже $p<0,02$, а эффективность дискриминации с применением простых классифицирующих функций без учета возможной когезивности наблюдений составила 62,5 \%, когда из множества фиксируемых в эти дни параметров были выделены следующие:

1) гемоглобин $-\mathrm{Hb}$, г/л;

2) гематокрит - Ht, \%;

3) эритроциты $-\operatorname{Er}\left(\times 10^{12} / \pi\right)$;

4) лейкоциты - Le $\left(\times 10^{9} / \pi\right)$;

5) $\mathrm{pH}$

6) $\mathrm{K}^{+}$, ммоль/л;

7) $\mathrm{Na}^{+}$, ммоль/л.

А по умершим, видимо, в силу того, что число наблюдений было невелико $(n=9)$, значимость уже первой дискриминантной функции оказалась на грани сомнения $(p<0,08)$ при эффективности дискриминации порядка 73,3\%.

Зависимости параметров в пространстве дискриминируемых переменных в целом и на межэтапном уровне представлены в табл. 4 и 5,

Таблица 4 / Table 4

Матрица корреляций параметров наблюдений по выжившим пациентам

Correlation matrix of observation parameters for surviving patients

\begin{tabular}{|l|c|c|c|c|c|c|c|}
\hline \multicolumn{1}{|c|}{$r^{\star} / r$} & $\mathrm{Hb}$ & $\mathrm{Ht}$ & $\mathrm{Er}$ & $\mathrm{Le}$ & $\mathrm{pH}$ & $\mathrm{K}^{+}$ & $\mathrm{Na}^{+}$ \\
\hline $\mathrm{Hb}$ & 1,000 & 0,951 & 0,910 & 0,077 & 0,285 & 0,052 & $-0,104$ \\
\hline $\mathrm{Ht}$ & 0,952 & 1,000 & 0,906 & 0,004 & 0,269 & 0,138 & $-0,124$ \\
\hline $\mathrm{Er}$ & 0,912 & 0,911 & 1,000 & 0,143 & 0,246 & 0,075 & 0,001 \\
\hline $\mathrm{Le}$ & 0,101 & 0,049 & 0,158 & 1,000 & $-0,160$ & $-0,147$ & $-0,024$ \\
\hline $\mathrm{pH}$ & 0,287 & 0,249 & 0,261 & $-0,041$ & 1,000 & 0,179 & $-0,115$ \\
\hline $\mathrm{K}^{+}$ & 0,039 & 0,110 & 0,063 & 0,001 & 0,084 & 1,000 & 0,220 \\
\hline $\mathrm{Na}^{+}$ & $-0,112$ & $-0,140$ & $-0,007$ & 0,021 & $-0,156$ & 0,154 & 1,000 \\
\hline
\end{tabular}

Примечание. Корреляции в целом представлены выше главной диагонали, а на межэтапном уровне - ниже главной диагонали.

Note. Correlations are generally shown above the main diagonal, and correlations at the inter-step level are shown below the main diagonal. 
Матрица корреляций параметров наблюдений по умершим пациентам

Таблица 5 / Table 5

Correlation matrix of observation parameters for deceased patients

\begin{tabular}{|l|c|c|c|c|c|c|c|}
\hline \multicolumn{1}{|c|}{$r^{\star} / r$} & $\mathrm{Hb}$ & $\mathrm{Ht}$ & $\mathrm{Er}$ & $\mathrm{Le}$ & $\mathrm{pH}$ & $\mathrm{K}^{+}$ & $\mathrm{Na}^{+}$ \\
\hline $\mathrm{Hb}$ & 1,000 & 0,917 & 0,897 & 0,049 & $-0,524$ & 0,135 & 0,017 \\
\hline $\mathrm{Ht}$ & 0,911 & 1,000 & 0,964 & $-0,013$ & $-0,514$ & 0,103 & $-0,172$ \\
\hline $\mathrm{Er}$ & 0,897 & 0,971 & 1,000 & 0,080 & $-0,474$ & 0,135 & $-0,284$ \\
\hline $\mathrm{Le}$ & 0,068 & 0,012 & 0,090 & 1,000 & $-0,292$ & 0,095 & $-0,254$ \\
\hline $\mathrm{pH}$ & $-0,481$ & $-0,462$ & $-0,448$ & $-0,357$ & 1,000 & 0,050 & $-0,116$ \\
\hline $\mathrm{K}^{+}$ & $-0,032$ & $-0,101$ & $-0,031$ & 0,155 & 0,365 & 1,000 & 0,063 \\
\hline $\mathrm{Na}^{+}$ & $-0,057$ & $-0,294$ & $-0,371$ & $-0,211$ & 0,000 & $-0,107$ & 1,000 \\
\hline
\end{tabular}

Примечание. Корреляции в целом представлены выше главной диагонали, а на межэтапном уровне - ниже главной диагонали.

Note. Correlations are generally shown above the main diagonal, and correlations at the inter-step level are shown below the main diagonal.

Таблица 6 / Table 6

Коэффициенты и свободный чмен канонической дискриминантной функции

Coefficients and free term of the canonical discriminant function

\begin{tabular}{|c|c|c|c|c|c|c|c|c|c|}
\hline \multirow{2}{*}{$\begin{array}{c}\text { № } \\
\text { дискриминантной } \\
\text { функции }\end{array}$} & \multicolumn{7}{|c|}{ Коэффициенты } \\
\cline { 2 - 10 } & $\mathrm{Hb}$ & $\mathrm{Ht}$ & $\mathrm{Er}$ & $\mathrm{Le}$ & $\mathrm{pH}$ & $\mathrm{K}^{+}$ & $\mathrm{Na}^{+}$ & $\begin{array}{c}\text { Свободный } \\
\text { член }\end{array}$ \\
\hline \multirow{3}{*}{ Выжившие } & $\mathrm{I}$ & 0,0134 & $-0,1096$ & 0,5492 & 0,0805 & $-6,4613$ & $-1,3883$ & $-0,0596$ & 59,3890 \\
\cline { 2 - 10 } & $\mathrm{II}$ & 0,0353 & $-0,3069$ & 1,6895 & 0,0184 & $-6,3416$ & 0,9125 & $-0,0275$ & 46,5570 \\
\cline { 2 - 10 } & III & 0,0089 & 0,3580 & $-3,6206$ & 0,1510 & 5,5303 & 0,3921 & 0,0383 & $-47,5585$ \\
\hline Умершие & I & $-0,0365$ & 0,1311 & $-0,2939$ & $-0,0244$ & $-3,9412$ & 0,6011 & 0,0202 & 25,1190 \\
\cline { 2 - 10 } & II & $-0,0312$ & $-0,3431$ & 3,9220 & $-0,0058$ & 0,6959 & 0,1220 & 0,0162 & $-9,5500$ \\
\cline { 2 - 10 } & III & $-0,0733$ & 0,0998 & 1,1944 & 0,0167 & 3,8030 & $-0,3028$ & 0,0540 & $-35,6184$ \\
\hline
\end{tabular}

Таблица 7 / Table 7

Коэффициенты и свободный чкен простых классифицирующих функций

Coefficients and free term of the simple classifying functions

\begin{tabular}{|c|c|c|c|c|c|c|c|c|c|}
\hline \multirow{2}{*}{\multicolumn{2}{|c|}{$\begin{array}{c}\text { День преодоления } \\
\text { политравмы }\end{array}$}} & \multicolumn{8}{|c|}{ Коэффициенты } \\
\hline & & \multirow{2}{*}{$\begin{array}{c}\mathrm{Hb} \\
-3,7011\end{array}$} & \multirow{2}{*}{$\begin{array}{c}\mathrm{Ht} \\
19,4723\end{array}$} & \multirow{2}{*}{$\begin{array}{c}\text { Er } \\
-86,4089\end{array}$} & \multirow{2}{*}{$\begin{array}{c}\text { Le } \\
2,0598\end{array}$} & \multirow{2}{*}{$\frac{\mathrm{pH}}{1396,5771}$} & \multirow{2}{*}{$\frac{\mathrm{K}^{+}}{-28,4189}$} & \multirow{2}{*}{$\begin{array}{c}\mathrm{Na}^{+} \\
11,6386\end{array}$} & \multirow{2}{*}{$\begin{array}{c}\text { Свободный } \\
\text { член }\end{array}$} \\
\hline Выжившие & 1-й & & & & & & & & \\
\hline & $2-и ̆$ & $-3,7346$ & 19,7049 & $-87,4543$ & 1,9857 & 1403,8010 & $-28,4015$ & 11,6846 & $-5896,1570$ \\
\hline & $3-и ̆$ & $-3,7085$ & 19,4952 & $-86,3408$ & 1,9494 & 1402,6369 & $-26,3711$ & 11,7048 & $-5897,5173$ \\
\hline & 4-й & $-3,7429$ & 19,8375 & $-88,3912$ & 1,9518 & 1409,1903 & $-27,3844$ & 11,7321 & $-5945,2284$ \\
\hline \multirow[t]{4}{*}{ Умершие } & 1-й & $-0,1438$ & 7,7553 & 66,8197 & 15,3466 & 1899,9542 & $-76,4786$ & 4,1757 & $-7610,8454$ \\
\hline & 2 -й & $-0,3015$ & 8,9336 & 60,0570 & 15,2464 & 1881,7619 & $-73,9289$ & 4,2702 & $-7490,2374$ \\
\hline & $3-и ̆$ & $-0,3677$ & 8,6545 & 63,9971 & 15,1638 & 1869,7852 & $-72,0231$ & 4,2930 & $-7411,4769$ \\
\hline & 4-й & $-0,4351$ & 8,4607 & 67,8547 & 15,2182 & 1880,8934 & $-73,2230$ & 4,3488 & $-7500,1449$ \\
\hline
\end{tabular}


а значения найденных при этом канонических дискриминантных и простых классифицирующих функций приведены соответственно в табл. 6 и 7.

Отметим, что коэффициенты простых классифицирующих функций по выжившим пациентам при тех же параметрах день ото дня разнятся не слишком, тогда как по умершим картина заметно контрастнее в проекции красной крови.

И чтобы полнее раскрыть роль отдельных параметров в достижении поэтапно существенных изменений состояния пациента, мы вычислили значения коэффициентов первой канонической дискриминантной функции при стандартизованных (приведенных к нулевой средней и к единичной дисперсии) переменных, а также получили соответствующие значения полных и группового уровня (в контексте «день ото дня») структурных коэффициентов (табл. 8, 9).

По выжившим (табл. 8) наиболее приоритетной среди других параметров, отражающих состояние ребенка, оказалась роль калия.

Хорошо известно, что политравма сопровождается быстрым развитием гипокалиемии и в силу того, что такая тенденция является стереотипной, иногда полагается $[15,16]$, что данная динамика не столь приоритетна как, скажем, проблема преодоления острой кровопотери, развития анемии. Однако по результатам проведенного дискриминантного анализа мы не можем полностью согласиться с вышеупомянутым тезисом. При этом весьма показательна в таком плане картина по погибшим пациентам (табл. 9), где критичность

Таблица 8 / Table 8

Коэффициенты, выражающие роль отАельных параметров наблюдений по выжившим пациентам

Coefficients that express the role of individual observation parameters for survivors

\begin{tabular}{|l|c|c|c|}
\hline \multicolumn{1}{|c|}{ Параметры } & $\begin{array}{c}\text { Коэффициенты первой канонической } \\
\text { дискриминантной функции } \\
\text { при стандартизованных параметрах }\end{array}$ & $\begin{array}{c}\text { Полные структурные } \\
\text { коэффициенты } \\
\text { дискриминации }\end{array}$ & $\begin{array}{c}\text { Структурные коэффициенты } \\
\text { дискриминации на срезе } \\
\text { «день ото дня» }\end{array}$ \\
\hline Гемоглобин & 0,1509 & $-0,072$ & $-0,054$ \\
\hline Гематокрит & $\mathbf{0 , 3 4 1 0}$ & $-0,173$ & $-0,127$ \\
\hline Эритроциты & 0,1960 & $-0,046$ & $-0,037$ \\
\hline Лейкоциты & 0,4054 & 0,593 & 0,444 \\
\hline рН & $-0,3274$ & $\mathbf{- 0 , 4 8 7}$ & $\mathbf{- 0 , 3 6 1}$ \\
\hline $\mathrm{K}^{+}$ & $-\mathbf{0 , 6 3 4 6}$ & $\mathbf{- 0 , 8 1 5}$ & $\mathbf{- 0 , 7 0 8}$ \\
\hline $\mathrm{Na}^{+}$ & $-0,1719$ & $-0,257$ & $-0,181$ \\
\hline
\end{tabular}

Примечание. Жирным шрифтом выделены требующие внимания величины.

Note. Important values are in bold.

Таблица 9 / Table 9

Коэффициенты, выражающие роль отАельных параметров наблюдений по умершим пациентам Coefficients that express the role of individual parameters of observations for deceased patients

\begin{tabular}{|l|c|c|c|}
\hline Параметры & $\begin{array}{c}\text { Коэффициенты первой канонической } \\
\text { дискриминантной функции } \\
\text { при стандартизованных параметрах }\end{array}$ & $\begin{array}{c}\text { Полные структурные } \\
\text { коэффициенты } \\
\text { дискриминации }\end{array}$ & $\begin{array}{c}\text { Структурные коэффициенты } \\
\text { дискриминации на срезе } \\
\text { «день ото дня» }\end{array}$ \\
\hline Гемоглобин & $\mathbf{0 , 7 9 3 5}$ & 0,317 & 0,133 \\
\hline Гематокрит & $\mathbf{0 , 9 9 8 6}$ & $\mathbf{0 , 3 6 8}$ & 0,141 \\
\hline Эритроциты & $-0,2886$ & 0,282 & 0,117 \\
\hline Лейкоциты & $-0,1656$ & $-0,116$ & $-0,013$ \\
\hline рН & $\mathbf{0 , 4 9 9 1}$ & $\mathbf{- 0 , 4 6 7}$ & $-0,160$ \\
\hline $\mathrm{K}^{+}$ & $\mathbf{0 , 7 5 8 2}$ & $\mathbf{0 , 7 0 7}$ & $\mathbf{0 , 4 3 4}$ \\
\hline $\mathrm{Na}^{+}$ & $\mathbf{0 , 3 6 1 6}$ & $\mathbf{0 , 3 8 8}$ & 0,145 \\
\hline
\end{tabular}

Примечание. Жирным шрифтом выделены требующие внимания величины.

Note. Important values are in bold. 
их состояния отмечена не только наивысшим приоритетом по калию, но еще и противоположными знаками роли гемоглобина и гематокрита. И хотя роль гематокрита и необходимость компенсаций по калию и натрию с учетом рН крови вполне отчетливо прослеживаются на уровне полных структурных коэффициентов дискриминации, все же на срезе «день ото дня» приоритетна лишь роль калия, что на фоне невысоких аналогичных коэффициентов по остальным параметрам следует, видимо, трактовать как свидетельство необратимости изменений в клетке при неутешительном в целом прогнозе для этих больных.

Вместе с тем следует отметить, что для выживших (табл. 8), и в целом, и с учетом особенных коррекций на срезе каждого дня, следующим после калия оказался приоритет роли лейкоцитов. Ведь хорошо известно, что на полученную травму организм обычно отвечает увеличением их числа за счет выхода из депо костного мозга, селезенки и в силу активации маргинального пула лейкоцитов. А далее в течение четырех суток наблюдаемое падение лейкоцитов связано в первую очередь с выходом клеточных элементов из крови в ткани, и только с пятых суток отмечается повышение, связанное уже с выходом в кровь клеток новой генерации [15]. Однако весьма настораживающим моментом в приведенных в табл. 9 выкладках по умершим пациентам оказывается тот факт, что значимой роли лейкоцитов у них не усматривается.

В целом же, по данным [16], в динамике таких показателей, как Hb, Ht и Er отмечается обычно рецессия к 3-му дню, увеличиваются они лишь с 5-го дня, и несомненно, что наиболее приоритетным является лечение анемии у пострадавших с политравмой с первых суток, ибо прогрессирующая анемия может быть основной причиной необратимых органных нарушений. Такой лечебный подход прописан в программе лечения тяжелого шока при политравме, целесообразность такого пути очевидна и не обсуждается [17]. Вполне очевидно, что и поддержание должного уровня гематокрита, как в не меньшей мере и $\mathrm{pH}$, оказываются важнейшими ориентирами при выведении пациента на траекторию исцеления.

\section{ВЫВО $\Delta$ Ы}

Таким образом, с применением дискриминантного анализа становится возможным полнее раскрыть особенности многомерной динамики проявлений политравмы у детей в первые дни реанимационных мероприятий и численно выразить приоритеты отражающих их состояние отдельных параметров. При этом, ориентируясь на расчетные величины канонических или простых классифицирующих функций, удается в целом более объективно оценивать состояние травмированного ребенка и по времени оперативно разграничивать индивидуальные особенности непрерывно меняющейся картины его дрейфа в танатогенном или же в реконвалесцентном направлении.

\section{АОПОАНИТЕАЬНАЯ ИНФОРМАЦИЯ}

Конфликт интересов. Авторы данной статьи подтвердили отсутствие конфликта интересов и финансовой поддержки, о которых необходимо сообщить.

Вклад в статью: В.Б. Баковский - написание раздела «введение», корректура статьи для издания; С.И. Головкин - постановка задачи, анализ научной литературы, описание результатов исследования, написание статьи; Н.А. Шабалдин - описание клинических наблюдений, перевод статьи на английский язык; Т.В. Кухарова - статистический анализ наблюдений и интерпретация его результатов, написание статьи; B.A. Уткин - анализ научной литературы, написание статьи, интерпретация результатов статистического анализа данных; Е.Н. Чалая - выбор метода анализа данных, методическое обеспечение исследования.

Conflict of interest. The authors of this article confirmed the lack of interest and financial support, which should be reported.

Information about the authors: V.B. $B a$ kovsky - contribution to the article: writing the "introduction" section, proofreading the article for publication; S.I. Golovkin - problem statement, analysis of scientific literature, description of research results, writing an article; N.A. Shabaldin - description of clinical observations, translation of the article into English; T.V. Kukharova - statistical analysis of observations and interpretation of its results, writing the article; V.A. Utkin - analysis of scientific literature, writing an article, interpretation of the results of statistical data analysis; E.N. Chalaya - choice of data analysis method, methodological support of the research. 


\section{АИТЕРАТУРА}

1. Талыпов А.Э., Мячин М.Ю., Куксова А.С., и др. Медикаментозная нейропротекция в остром периоде черепно-мозговой травмы средней степени тяжести // Медицинский Совет. - 2015. - № 10. - C. 82-83. [Talypov AE, Myatchin MYu, Kuksova NS, et al. Drug-based neuroprotection in acute phase of traumatic brain injury of moderate severity. Medical Council. 2015;(10):82-83. (In Russ.)]. DOI: https://doi. org/10.21518/2079-701X-2015-10-82-92

2. Peterson N, James L. Polytrauma in children. Orthopaedics and Trauma. 2018;32(5):280-287. DOI: https://doi.org/10.1016/j.mporth.2018.07.004

3. Пшениснов К.В., Александрович Ю.С., Миронов П.И., и др. Особенности инфузионной терапии у детей с тяжелой сочетанной травмой // Анестезиология и реаниматология. 2016. - № 1. - C. 28-32. [Pshenisnov KV, Aleksandrovich YuS, Mironov PI, et al. Features of fluid therapy in children with severe major trauma. Anesthesiology and Intensive Care. 2016;(1):28-32. (In Russ.)] DOI: https://doi.org/10.18821/02017563-2016-61-1-28-32.)

4. Naqvi G, Johansson G, Yip G, et al. Mechanisms, Patterns and outcomes of paediatric polytrauma in a UK major trauma centre. Ann $R$ Coll Surg. 2017;99(1):39-45. DOI: https://doi.org/10.1308/ rcsann.2016.0222.

5. Тимофеев В.В., Бондаренко А.В. Структура и характер политравмы у детей в городе Барнауле // Травматология и ортопедия России. - 2013. - № 2. - C. 94-98. [Timofeev VV, Bondarenko AV. The structure and feature of polytrauma in children of Barnaul. Traumatology and Orthopedics of Russia. 2013;(2):94-98. (In Russ.)] DOI: https://doi.org/10.21823/23112905-2013-2-94-98.

6. Баковский В.Б., Гибадуллин Д.Г., Шабалдин Н.А., и др. Структурирование политравмы у детей с применением оценки по системе ISS и концентрации маркеров белка s100b и цистатина C// Российский Вестник детской хирургии анестезиологии и реаниматологии. 2019. - T. 9. - № 1. - C. 70-77. [Bakowski VB, Gibadyllin DG, Shabaldin NA, et al. Multiple trauma structuring in children with estimation according to iss system and concentrations of S100B protein markers and cystatin C. Russian Journal of Pediatric Surgery, Anesthesia and Intensive Care. 2019;9(1):70-77. (In Russ.)] DOI: https://doi.org/10.30946/2219-4061-2019-91-70-77.

7. Karlbauer A, Woidke R. Оценка тяжести травмы: обзор наиболее часто используемых систем для оценки тяжести повреждений у травматологических больных// Вестник травматологии и ортопедии им. Н.Н. Приорова. - 2003. - № 3. - C. 16-19. [Karlbauer A, Woidke R. Evaluation of Injury Severity: Review of the Most Commonly Used System for the
Evaluation of Injury Severity in Traumatologic Patients. N.N. Priorov Journal of Traumatology and Orthopedics. 2003;(3):16-19. (In Russ.)]

8. Уткин В.А., Головкин С.И., Першин И.М., и др. Пограничные и терминальные состояния в свете теории управления // ХX Юбилейная Всероссийская научно-практическая конференция «Интегративная медицина. 10 лет журналу «Политравма»: 9 мая 2017 г.: сборник тезисов - Ленинск-Кузнецкий. 2017. - C. 104-105. [Utkin VA, Golovkin SI, Pershin IM, et al. Boundary and terminal States in the light of control theory. XX anniversary allRussian scientific-practical conference "Integrative medicine. 10 years of the journal Polytrauma": may 9, 2017: abstracts - Leninsk-Kuznetsk. 2017. P. 104-105. (In Russ.)]

9. Уткин В.А., Першин И.М., Головкин С.И., и др. Особенности идентификации динамики метаболизма в живой системе исходя из канонов медицинских классификаций // VIII Всероссийская научная конференция "Системный синтез и прикладная синергетика»: сборник научных трудов. - Ростов н/Д; Таганрог: Издательство Южного федерального университета. - 2017. - C. 391-396. [Utkin VA, Pershin IM, Golovkin SI, et al. Features of identification of the dynamics of metabolism in a living system based on the canons of medical classifications. VIII all-Russian scientific conference "System synthesis and applied synergetics": collection of scientific works; Rostov-on-Don; Taganrog: Southern Federal University Press, 2017. P. 391396. (In Russ.))

10. Ким Дж.-О., Мьюллер Ч.У., Клекка У.Р., и др. Факторный, дискриминантный и кластерный анализ / пер. с англ.; под. ред. И.С. Енюкова. - М.: Финансы и статистика, 1989. - 215 с. [Kim J-O, Muller ChW, Klekka WR, et al. Factor, discriminant and cluster analysis: Transl. eng.; Under. edited by I.S. Eniukov; Moscow: Finance and statistics, 1989. 215 p. (In Russ.)]

11. Уткин В.А. Статистические технологии в медицинских исследованиях: Монография, 2-е изд., испр., доп. - Пятигорск: ГНИИК ФМБА РФ, 2012. - 212 c. [Utkin VA. Statistical technologies in medical research: Monography, $2^{\text {nd }}$ ed., Rev. extra; Piatigorsky: GNIIK FMBA of Russia. 2012. 212 p. (In Russ.)]

12. Кухарова Т.В., Папуш Е.Г., Першин И.М., Уткин В.А. Управляющие воздействия для объектов, описываемых дискриминантными или регрессионными функциями // Современная наука и инновации. - 2016. - Т. 16. № 4. - C. 29-33. [Kukharova TV, Papush EG, Pershin IM, Utkin VA. Control actions for the objects described by discriminant or regression functions. Modern science and innovations. 2016;16(4):29-33. (In Russ.)]

13. Уткин В.А. Методические аспекты математико-статистического анализа медицинских данных Часть 3. Классификация и диагности- 
ка в современной медицине с применением дискриминантного и кластерного анализа // Медицинский вестник Северного Кавказа. 2009. - T. 16. - № 4. - C. 62-65. [Utkin VA. Methodological aspects of mathematical and statistical analysis of medical data Part 3 . Classification and diagnostics in modern medicine using discriminant and cluster analysis. Medical Bulletin of the North Caucasus. 2009;16(4):62-65. (In Russ.)]

14. Гайдышев И.П. Анализ и обработка данных: Специальный справочник. - СПб.: Питер, 2001. 752 c. [Gaidyshev IP. Analysis and data processing: Special directory. Saint Petersburg: Peter, 2001. 752 p.]

15. Оценка лабораторных данных в критических состояниях (трактовка, прогнозирование, медикаментозная коррекция) / под ред. проф. С.И. Перегудова. - СПб., 1999. - 76 с. [Assessment of laboratory data in critical conditions (interpretation, prediction, medicamental correction) under the editorship of Professor S.I. Peregudova; Saint Petersburg, 1999. 76 p. (In Russ.)]

16. Устьянцева И.М. Лабораторная диагностика при политравме // Политравма. - 2008. № 4. - C. 51-54. [Ustyantseva IM. Laboratory diagnosis in polytrauma. Polytrauma. 2008;(4):5154. (In Russ.)]

17. Агаджанян В.В., Устьянцева И.М., Хохлова О.И. Синдром системного воспалительного ответа и полиорганная дисфункция у детей с политравмой // Политравма. - 2012. - № 4: C. 69-81. [Agadzhanyan VV, Ustyantseva IM, Khokhlova OI. Systemic inflammatory response Syndrome and multiple organ dysfunction in children with polytrauma. Polytrauma. 2012;(4):69-81. (In Russ.)]

\section{Информачия об авторах}

Владислав Богданович Баковский - канд. мед. наук, врач, отделение травматологии-ортопедии. ГАУЗ КО «Областная детская клиническая больница», Кемерово. E-mail: bakovskivb@rambler.ru.

Сергей Иванович Головкин - д-р мед. наук, доцент, профессор кафедры детских хирургических болезней. ФГБОУ ВО КемГМУ Минздрава России, Кемерово. E-mail: golovkins2@mail.ru.

Татьяна Валерьевна Кухарова - канд. тех. наук, доцент кафедры медицинской радиоэлектроники. СанктПетербургский государственный университет аэрокосмического приборостроения, Санкт-Петербург. E-mail: unit-4@yandex.ru.

Владимир Александрович Уткин - д-р мед. наук, ведущий научный сотрудник. ПГНИИК филиал ФФГБУ СКФНКЦ ФМБА России, г. Пятигорск. E-mail: orgotdel@ gniik.ru.

Елена Николаевна Чалая - канд. мед. наук, доцент, ученый секретарь. ПГНИИК филиал ФФГБУ СКФНКЦ ФМБА России, Пятигорск. E-mail: orgotdel@gniik.ru.

Никита Андреевич Шабалдин - канд. мед. наук, доцент кафедры детских хирургических болезней. ФГБОУ BO КемГМУ Минздрава России, Кемерово. E-mail: shabaldin.nk@yandex.ru.
Information about the authors

Vladislav B. Bakovsky - Cand. Sci. (Med.), doctor, Department of traumatology and orthopedics. Regional Children's Clinical Hospital, Kemerovo, Russia. E-mail: bakovskivb@rambler.ru.

Sergey I. Golovkin - Dr. Sci. (Med.), associate Professor, Professor of the Department of pediatric surgical diseases. KemSMU of the Ministry of Health of the Russian Federation, Kemerovo, Russia. E-mail: golovkins2@mail.ru.

Tatyana V. Kukharova - Cand. Sci. (Tech.), associate Professor of the Department of medical Radioelectronics. Saint Petersburg State University of Aerospace Instrumentation, Saint Petersburg, Russia. E-mail: unit-4@yandex.ru.

Vladimir A. Utkin - Dr. Sci. (Med.), leading researcher. PGNIIK branch of the Federal State Budgetary Institution of the Russian Federation, Pyatigorsk, Russia. E-mail: orgotdel@gniik.ru.

Elena N. Chalaya - Cand. Sci. (Med.), scientific Secretary. PGNIIK branch of the Federal State Budgetary Institution SKFNKC FMBA of Russia, Pyatigorsk, Russia. E-mail: orgotdel@gniik.ru.

Nikita A. Shabaldin - Cand. Sci. (Med.), associate Professor of the Department of pediatric surgical diseases. KemSMU of the Ministry of Health of the Russian Federation, Kemerovo, Russia. E-mail: shabaldin.nk@yandex.ru. 